\title{
Input-output LPV model identification with guaranteed quadratic stability
}

\author{
V. Cerone ${ }^{*}$, D. Piga ${ }^{* *}$, D. Regruto ${ }^{*}$, R. Tóth ${ }^{* *}$ \\ * Dipartimento di Automatica e Informatica, Politecnico di Torino, Corso \\ Duca degli Abruzzi 24, 10129 Torino, Italy, \\ e-mail: vito.cerone@polito.it, diego.regruto@polito.it \\ ** Delft Center for Systems and Control, Delft University of Technology, \\ Mekelweg 2, Delft, 2628 CD, The Netherlands, \\ e-mail:D.Piga@tudelft.nl,R.Toth@tudelft.nl
}

\begin{abstract}
The problem of identifying linear parameter-varying (LPV) systems, a priori known to be quadratically stable, is considered in the paper using an input-output model structure. To solve this problem, a novel constrained optimization-based algorithm is proposed which guarantees quadratic stability of the identified model. It is shown that this estimation objective corresponds to a nonconvex optimization problem, defined by a set of polynomial matrix inequalities (PMI), whose optimal solution can be approximated by means of suitable convex semidefinite relaxations. Applicability of such relaxation-based estimation approach in the presence of either stochastic or deterministic bounded noise is discussed. A simulation example is also given to demonstrate the effectiveness of the resulting identification method.
\end{abstract}

Keywords: LPV system; quadratic stability; polynomial optimization; convex relaxation.

\section{INTRODUCTION}

Linear parameter-varying (LPV) models are linear systems whose parameters depend on a time-varying, possibly vectorvalued, exogenous variable, whose real-time measurements are assumed to be available. Due to recent developments in LPV modeling and control, the LPV modeling paradigm can be considered as one of the effective tools to derive a mathematical description of nonlinear/time-varying phenomena and synthesize efficient controllers for such systems. Particularly, a rapidly increasing number of contributions addressing the problem of black-box LPV model identification from input-output measurements has recently appeared in the literature, including recursive (Bamieh and Giarré (2002)) and separable (Previdi and Lovera (2004)) least squares algorithms, subspace identification (Verdult and Verhaegen (2002, 2005); Felici et al. (2007); Lopes dos Santos et al. (2008)) and orthonormal basis functions (Tóth et al. (2010)) approaches, instrumental-variables-based algorithms (Laurain et al. (2010)), set-membership methods (Cerone and Regruto (2008); Cerone et al. (2011a)) and many more. A detailed overview of the available LPV modeling and identification approaches can be found in the recent book by Tóth (2010).

Most of the identification algorithms available in the literature rely on input-output data collected from open-loop experiments, which can be performed in practice only if the system to be identified is a-priori known to be stable. A number of strategies to enforce stability constraints in the identification of linear time-invariant (LTI) systems can be found in the literature, however such developments have not been achieved in the LPV framework yet. In the LTI case, a sufficient condition to ensure stability of dynamic models obtained by least squares (LS) identification is provided in Regalia and Stoica (1995), where the input signal is constrained to be an autoregressive process with a specific degree. Tugnait and Tontiruttananon (1998) provide a frequency-domain solution to LS identification of a stable system in the presence of undermodeling. The proposed approach is applicable when the input signal is a zero-mean stationary process with sufficiently high order of persistency of excitation. In (Janakiraman and Bhattacharyya (1999)), a stable output-error identification scheme is presented for the specific case of all-pole systems and periodic excitation signals, while Nicolao and Pillonetto (2008) give a procedure to include prior information on BIBO stability in the context of kernel-based nonparametric identification. Cerone et al. (2011b) describe a method to enforce stability constraints in evaluating bounds on the parameters of LTI transfer functions identified from input and output data affected by bounded measurement noise. Different approaches are also available in the literature to enforce stability in LTI subspace identification. The interested reader can refer to (Lacy and Bernstein (2003)) and the references therein for a thorough review on the subject. The most recent and effective method among such approaches is the one proposed by Lacy and Bernstein (2003) where prior information on asymptotic stability is directly taken into account in the computation of the LS estimate through the solution of a proper convex optimization problem.

To the best of the authors' knowledge, no contribution can be found in the literature that formally include mathematical constraints related to stability in the context of LPV model identification. Consequently, the identification procedure may give rise to inaccurate and possibly unstable models, especially in the presence of relatively short data records, structural modeling error and significant measurement noise. 
In this work, an original approach is proposed to enforce quadratic stability constraints in the identification of inputoutput LPV models with affine dependence on the scheduling variables. The paper is organized as follows. Section 2 is devoted to the formulation of the assumed identification problem. Previous results on quadratic stability of input-output LPV models are briefly reviewed in Section 3. An algorithm for the solution of the considered problem is then proposed in Section 4 under the assumption that the scheduling variables range inside a polytope. In this case, the problem is shown to be equivalent to a nonconvex polynomial matrix inequality (PMI) problem whose global optimum can be arbitrarily well approximated by exploiting suitable convex relaxation techniques. Effectiveness of the proposed approach is shown by means of a simulation example in Section 5. Concluding remarks are presented at the end of the paper.

\section{PROBLEM FORMULATION}

Consider a SISO, discrete-time LPV ARX model described in terms of the following linear difference equations

$$
\begin{aligned}
\mathcal{A}\left(q^{-1}, \lambda(t)\right) w(t) & =\mathcal{B}\left(q^{-1}, \lambda(t)\right) u(t), \\
\mathcal{A}\left(q^{-1}, \lambda(t)\right) \eta(t) & =e(t), \\
y(t) & =w(t)+\eta(t),
\end{aligned}
$$

where $q^{-1}$ is the backward time-shift operator, i.e., $q^{-1} w(t)=$ $w(t-1), u(t): \mathbb{Z} \rightarrow \mathbb{R}$ is the input signal, $w(t): \mathbb{Z} \rightarrow \mathbb{R}$ is the noise-free output signal, $y(t): \mathbb{Z} \rightarrow \mathbb{R}$ is the measured output signal, $e(t): \mathbb{Z} \rightarrow \mathbb{R}$ is a white noise process, $\eta(t)$ : $\mathbb{Z} \rightarrow \mathbb{R}$ is the effect of the noise $e(t)$ on the measured output signal, $\lambda(t): \mathbb{Z} \rightarrow \mathbb{R}^{\mu}$ is the scheduling variable which, according to the LPV modeling and control literature (see, e.g., Rugh and Shamma (2000)) is assumed to be measurable. The scheduling variable $\lambda(t)$ is assumed to belong to a compact set $\Lambda \subset \mathbb{R}^{\mu}$. In order to simplify notation, in the rest of the paper the following shorthand notation will be adopted for a generic signal $\pi_{t} \triangleq \pi(t)$. $\mathcal{A}(\cdot)$ and $\mathcal{B}(\cdot)$ are polynomials in the backward shift operator $q^{-1}$ described as

$$
\begin{aligned}
& \mathcal{A}\left(q^{-1}, \lambda_{t}\right)=1+a_{1}\left(\lambda_{t}\right) q^{-1}+\ldots+a_{n_{\mathrm{a}}}\left(\lambda_{t}\right) q^{-n_{\mathrm{a}}} \\
& \mathcal{B}\left(q^{-1}, \lambda_{t}\right)=b_{0}\left(\lambda_{t}\right)+b_{1}\left(\lambda_{t}\right) q^{-1}+\ldots+b_{n_{\mathrm{b}}}\left(\lambda_{t}\right) q^{-n_{\mathrm{b}}}
\end{aligned}
$$

where $n_{\mathrm{a}}, n_{\mathrm{b}} \geq 0$ and the coefficients $a_{i}$ and $b_{j}$ are assumed to be affine functions of $\lambda_{t}=\left[\begin{array}{llll}\lambda_{t, 1} & \lambda_{t, 2} & \ldots & \lambda_{t, \mu}\end{array}\right]$ parameterized as follows

$$
\begin{aligned}
& a_{i}\left(\lambda_{t}\right)=a_{(i, 0)}+\sum_{\gamma=1}^{\mu} a_{(i, \gamma)} \lambda_{t, \gamma}, \\
& b_{j}\left(\lambda_{t}\right)=b_{(j, 0)}+\sum_{\nu=1}^{\mu} b_{(j, \nu)} \lambda_{t, \nu} .
\end{aligned}
$$

The unknown parameter vector $\theta \in \mathbb{R}^{n_{\theta}}, n_{\theta}=(\mu+1)\left(n_{\mathrm{a}}+\right.$ $\left.n_{\mathrm{b}}+1\right)$ to be estimated is defined as

$$
\begin{aligned}
\theta=\left[\begin{array}{lllllllll}
a_{1,0} & a_{1,1} & \ldots & a_{1, \mu} & \ldots & a_{n_{\mathrm{a}}, 0} & a_{n_{\mathrm{a}}, 1} & a_{n_{\mathrm{a}}, \mu} \\
b_{0,0} & b_{0,1} & \ldots & b_{0, \mu} & \ldots & b_{n_{\mathrm{b}}, 0} & b_{n_{\mathrm{b}}, 1} & \ldots & b_{n_{\mathrm{b}}, \mu}
\end{array}\right] .
\end{aligned}
$$

The parameter vector associated with the true-data-generating system is denoted by $\theta_{0}$.

In this paper, we address the problem of identifying LPV systems that are a-priori known to be quadratically stable in the set $\Lambda$. The notion of quadratic stability of a LPV system in an input-output form is introduced by the following definition (see also Gilbert et al. (2010) and Tóth (2010)).
Definition 1. (Quadratic stability of LPV systems given an IO representation)

Let us define

$$
x(t)=\left[y(t) y(t-1) \ldots y\left(t-n_{\mathrm{a}}+1\right)\right]^{\top}
$$

which can be considered as a state variable for the system in (1a). The LPV system (1a) is quadratically stable, if and only if there exists a symmetric matrix $P=P^{\top} \succ 0$, such that the quadratic function

$$
V(t)=x(t)^{\top} P x(t),
$$

is a Lyapunov function which implies Lyapunov stability of (1a) for all possible trajectories of $\lambda_{t}$ inside the compact set $\Lambda \subset \mathbb{R}^{\mu}$, i.e. $\forall \lambda \in \Lambda^{\mathbb{Z}}$.

In order to explicitly take into account prior information on quadratic stability, the following identification problem is considered:

$$
\tilde{\theta}=\arg \min _{\theta \in \mathcal{D}_{\theta}^{\mathrm{qs}}} J(\theta, \bar{u}, \bar{y})
$$

where $\mathcal{D}_{\theta}^{\mathrm{qs}} \subset \mathbb{R}^{n_{\theta}}$ is the set of all $\theta$ for which the LPV system is quadratically stable in the sense of Definition $1, \bar{u}, \bar{y}, \bar{\lambda}$ are the collected input, output and scheduling variables sequences respectively, and $J(\theta, \bar{u}, \bar{y})$ is assumed to be a generic multivariate polynomial function of parameters $\theta$. This choice for $J(\theta, \bar{u}, \bar{y})$ is quite general and allows us to consider most common identification criteria, including, for example, the leastsquares functional

$$
J=\|\bar{y}-\Phi \theta\|_{2}^{2}
$$

and the $L_{1}$-regularized least squares functional

$$
J=\|\bar{y}-\Phi \theta\|_{2}^{2}+\alpha\|\theta\|_{1}
$$

where

$$
\bar{y}=[y(1) y(2) \ldots y(N)]^{\top}, \Phi=[\phi(1) \phi(2) \ldots \phi(N)]^{\top}
$$

and

$$
\begin{aligned}
\phi(t)=\left[\begin{array}{lll}
-y(t-1) & -\lambda(t) y(t-1) & \ldots \\
& -y\left(t-n_{\mathrm{a}}\right) & -\lambda(t) y\left(t-n_{\mathrm{a}}\right) \\
& u(t) \quad \lambda(t) u(t) \quad \ldots \\
& u\left(t-n_{\mathrm{b}}\right) \quad \lambda(t) u\left(t-n_{\mathrm{b}}\right)
\end{array}\right]^{\top} .
\end{aligned}
$$

Our objective is to present an approach to enforce quadratic stability constraints in the identification of input-output LPV systems. Because such constraints are independent from the noise model, the presented approach can be applied to problems with different structures of noise. For the sake of simplicity, in the sequel we restrict ourself to the ARX case.

\section{QUADRATIC STABILITY OF LPV SYSTEMS IN INPUT-OUTPUT FORM}

The aim of this section is to briefly summarize some results on quadratic stability via input-output formulation of LPV systems, originally reported in Gilbert et al. (2010), that will be used in the rest of the paper to solve problem (7).

Let us define the following quantities

$$
\begin{aligned}
& \bar{a}=\left[a_{n_{\mathrm{a}}}(\lambda) \ldots a_{2}(\lambda) a_{1}(\lambda) 1\right],
\end{aligned}
$$

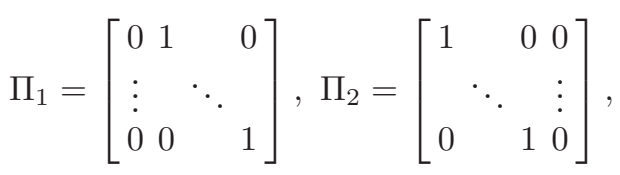


with $\Pi_{1}, \Pi_{2} \in \mathbb{R}^{n_{\mathrm{a}} \times n_{\mathrm{a}+1}}$. The following theorem provides a sufficient condition for quadratic stability of LPV systems described by (1a).

Theorem 1. (Gilbert et al. (2010))

Given a polynomial $\mathcal{C}\left(q^{-1}\right)$ of order $n_{\mathrm{a}}$

$$
\mathcal{C}\left(q^{-1}\right)=1+c_{1} q^{-1}+\ldots+c_{n_{\mathrm{a}}} q^{-n_{\mathrm{a}}},
$$

with roots inside the unite circle, the LPV system in (1a) is quadratically stable in $\Lambda$, if there exists a symmetric matrix $P \in \mathbb{R}^{n_{\mathrm{a}} \times n_{\mathrm{a}}}$ such that

$$
\bar{c}^{\top} \bar{a}(\lambda)+\bar{a}^{\top}(\lambda) \bar{c}-F(P) \succeq 0, \forall \lambda \in \Lambda
$$

where $\bar{c}=\left[\begin{array}{lllll}c_{n_{\mathrm{a}}} & \ldots & c_{2} & c_{1} & 1\end{array}\right]$ and

$$
F(P)=\Pi_{1}^{\top} P \Pi_{1}-\Pi_{2}^{\top} P \Pi_{2} \text {. }
$$

The polynomial $\mathcal{C}\left(q^{-1}\right)$ given in Theorem 1 is referred to as the stable central polynomial.

Remark 1. It is worth noting that, for given constant polynomial coefficients $\bar{c}$, condition (15) is a linear matrix inequality (LMI) constraint in $\theta$ and in the entries of the matrix $P$, polynomially parameterized in the scheduling variable $\lambda$. Therefore, (15) leads to a semi-infinite LMI constraint.

Remark 2. In Gilbert et al. (2010), condition (15) is used to design a robust controller under the quite general assumption that $\Lambda$ is a semialgebraic set. After selecting a stable polynomial $\mathcal{C}\left(q^{-1}\right)$, the problem of computing a vector $\bar{a}(\lambda)$ satisfying the constraint $\bar{c}^{\top} \bar{a}(\lambda)+\bar{a}(\lambda)^{\top} \bar{c}-F(P) \succeq 0$ for all $\lambda$ belonging to $\Lambda$ is solved in terms of sum-of-squares matrix polynomial optimization (see Gilbert et al. (2010); Scherer (2006) and references therein). Unfortunately, as discussed in Henrion et al. (2003), deriving a general formula for the selection of the central polynomial is a hard task (see also Gilbert et al. (2010) for a discussion on this point).

\section{LPV MODEL IDENTIFICATION WITH GUARANTEED QUADRATIC STABILITY}

Let us denote with $\widetilde{\mathcal{D}}_{\theta}^{\text {qs }}$ the set of all $\theta$ that satisfy equation (15) for a given stable polynomial $\mathcal{C}\left(q^{-1}\right)$. It is worth noting that $\widetilde{\mathcal{D}}_{\theta}^{\mathrm{qs}}$ is an inner approximation of $\mathcal{D}_{\theta}^{\mathrm{qs}}$. The degree of this approximation strongly depends on the selected central polynomial. Therefore, for a given stable polynomial $\mathcal{C}_{*}\left(q^{-1}\right)=1+$ $\bar{c}_{* 1} q^{-1}+\ldots+\bar{c}_{* n_{\mathrm{a}}} q^{-n_{\mathrm{a}}}$ with order $n_{\mathrm{a}}$, a suboptimal solution $\breve{\theta}$ to problem (7) can be computed as follows:

$$
\begin{aligned}
\breve{\theta}= & \arg \min _{\theta, P} J(\theta, \bar{u}, \bar{y}) \\
& \text { s.t. } \bar{c}_{*}^{\top} \bar{a}(\lambda)+\bar{a}(\lambda)^{\top} \bar{c}_{*}-F(P) \succeq 0, \forall \lambda \in \Lambda,
\end{aligned}
$$

where $\bar{c}_{*}=\left[\bar{c}_{* n_{\mathrm{a}}}, \ldots, \bar{c}_{* 1}, 1\right]$.

Since the level of suboptimality of $\breve{\theta}$ strongly depends on the choice of the central polynomial, we propose to modify problem (17) as follows

$$
\begin{aligned}
\hat{\theta}= & \arg \min _{\theta, P, \bar{c}} J(\theta, \bar{u}, \bar{y}) \\
\text { s.t. } & \bar{c}^{\top} \bar{a}(\lambda)+\bar{a}(\lambda)^{\top} \bar{c}-F(P) \succeq 0, \forall \lambda \\
& \bar{c} \in \mathcal{S}_{c}
\end{aligned}
$$

where $\mathcal{S}_{c}$ is the set of all stable polynomial of order $n_{\mathrm{a}}$, and the functional $J$ is now also minimized with respect to the coefficients of the central polynomial $\mathcal{C}\left(q^{-1}\right) \in \mathcal{S}_{c}$. As pointed out in Cerone et al. (2011b), the region $\mathcal{S}_{c}$ can be described
Table 1. Jury's array.

\begin{tabular}{ccclccc}
\hline$c_{n_{\mathrm{a}}}$ & $c_{n_{\mathrm{a}}-1}$ & $c_{n_{\mathrm{a}}-2}$ & $\cdots$ & $c_{2}$ & $c_{1}$ & 1 \\
1 & $c_{1}$ & $c_{2}$ & $\cdots$ & $c_{n_{\mathrm{a}}-2}$ & $c_{n_{\mathrm{a}}-1}$ & $c_{n_{\mathrm{a}}}$ \\
$r_{n_{\mathrm{a}}-1}$ & $r_{n_{\mathrm{a}}-2}$ & $r_{n_{\mathrm{a}}-3}$ & $\cdots$ & $r_{1}$ & $r_{0}$ & \\
$r_{0}$ & $r_{1}$ & $r_{2}$ & $\cdots$ & $r_{n_{\mathrm{a}}-2}$ & $r_{n_{\mathrm{a}}-1}$ & \\
$s_{n_{\mathrm{a}}-2}$ & $s_{n_{\mathrm{a}}-3}$ & $s_{n_{\mathrm{a}}-4}$ & $\cdots$ & $s_{0}$ & & \\
$\vdots$ & $\vdots$ & $\vdots$ & $\vdots$ & & & \\
$q_{2}$ & $q_{1}$ & $q_{0}$ & & & & \\
\hline
\end{tabular}

by means of a set of scalar polynomial inequalities in the coefficients of $\mathcal{C}_{*}\left(q^{-1}\right)$. In particular, the following result holds.

Result 1. (Cerone et al. (2011b))

$\mathcal{S}_{c}$ is a semialgebraic set defined by the following polynomial inequalities in the coefficients $c_{1}, \ldots, c_{n_{\mathrm{a}}}$

$$
\begin{gathered}
\mathcal{C}(1)>0, \quad(-1)^{n_{\mathrm{a}}} \mathcal{C}(-1)>0, \quad\left|c_{n_{\mathrm{a}}}\right|<1, \\
r_{n_{\mathrm{a}}-1}^{2}<r_{0}^{2}, \quad s_{n_{\mathrm{a}}-2}^{2}<s_{0}^{2}, \quad \ldots, \quad q_{2}^{2}<q_{0}^{2},
\end{gathered}
$$

where $r_{0}, s_{0}, \ldots, q_{0}, \ldots, r_{n_{\mathrm{a}}-1}, s_{n_{\mathrm{a}}-2}, \ldots, q_{2}, q_{1}$ are elements of the Jury's array reported in Table 1, i.e. $r_{n_{\mathrm{a}}-j_{\mathrm{c}}}=$ $\operatorname{det}\left(\begin{array}{cc}c_{n_{\mathrm{a}}} & c_{n_{\mathrm{a}}-j_{\mathrm{c}}} \\ 1 & c_{j_{\mathrm{c}}}\end{array}\right), s_{n_{\mathrm{a}}-j_{\mathrm{d}}}=\operatorname{det}\left(\begin{array}{cc}r_{n_{\mathrm{a}}-1} & r_{n_{\mathrm{a}}-j_{\mathrm{d}}} \\ r_{0} & r_{j_{\mathrm{d}}}\end{array}\right)$, with $c_{0}=$ 1 and $\operatorname{det}(\cdot)$ denoting the determinant of a matrix.

Since the constraint $\bar{c}^{\top} \bar{a}(\lambda)+\bar{a}(\lambda)^{\top} \bar{c}-F(P) \succeq 0$ is a nonconvex bilinear matrix inequality (BMI) in $\bar{c}$ and $\theta$, polynomially parameterized in $\lambda$, problem (18) is a semi-infinite nonconvex polynomial matrix inequalities (PMI) problem which is difficult to solve in general. Here we propose an algorithm for the solution of problem (18) under the following additional assumption. Assumption 1. $\Lambda$ is a polytope with vertices $\lambda_{1}^{v}, \lambda_{2}^{v}, \ldots, \lambda_{\rho}^{v}$.

Assumption 1 leads to the following reformulation of Theorem 1.

Theorem 2.

Given a stable polynomial $\mathcal{C}\left(q^{-1}\right) \in \mathcal{S}_{c}$ of order $n_{\mathrm{a}}$, the LPV system in (1a), satisfying Assumption 1, is quadratically stable in $\Lambda$ if there exists a symmetric matrix $P \in \mathbb{R}^{n_{\mathrm{a}} \times n_{\mathrm{a}}}$ such that

$$
\bar{c}^{\top} \bar{a}\left(\lambda_{i}^{v}\right)+\bar{a}\left(\lambda_{i}^{v}\right)^{\top} \bar{c}-F(P) \succeq 0, \forall i=1,2, \ldots, \rho
$$

Proof We have to prove that, under Assumption 1, condition (21) is a necessary and sufficient condition for (15). Necessity is straightforward, since condition (21) is satisfied by construction when (15) holds. In order to prove sufficiency, let us define the following function

$$
\chi(w, \lambda)=w^{\top}\left[\bar{c}^{\top} \bar{a}(\lambda)+\bar{a}^{\top}(\lambda) \bar{c}-F(P)\right] w
$$

where $w$ is any nonzero vector such that $w \in \mathbb{R}^{n_{\mathrm{a}}}$ and $P$ is a symmetric matrix such that

$$
\bar{c}^{\top} \bar{a}(\lambda)+\bar{a}^{\top}(\lambda) \bar{c}-F(P) \succeq 0, \forall \lambda \in \Lambda .
$$

As is well known, condition (23) is equivalent to

$$
\chi(w, \lambda) \geq 0, \forall \lambda \in \Lambda, \forall w \in \mathbb{R}^{n_{\mathrm{a}}}, w \neq 0 .
$$

Due to the affine dependence of $\bar{a}(\lambda)$ on $\lambda$, the minimum of function $\chi(w, \lambda)$ over the polytope $\Lambda$ is attained on one of the vertexes $\lambda_{1}^{v}, \lambda_{2}^{v}, \ldots, \lambda_{\rho}^{v}$, being $\chi(w, \lambda)$ a linear functional of $\lambda$. Proof of sufficiency follows from the fact that

$$
\chi\left(w, \lambda_{i}^{v}\right) \geq 0 \forall i=1, \ldots, \rho,
$$

thanks to condition (21).

Due to Theorem (2), problem (18) simplifies to the following finite dimensional nonconvex PMI problem 


$$
\begin{aligned}
& \hat{\theta}=\arg \min _{\theta, P, \bar{c}} J(\theta, \bar{u}, \bar{y}) \\
& \text { s.t. } \bar{c}^{\top} \bar{a}\left(\lambda_{i}^{v}\right)+\bar{a}\left(\lambda_{i}^{v}\right)^{\top} \bar{c}-F(P) \succeq 0, \forall i=1,2, \ldots, \rho \\
& \quad C \in \mathcal{S}_{c} .
\end{aligned}
$$

Remark 3. Since $e(t): \mathbb{Z} \rightarrow \mathbb{R}$ is a white noise process, consistency of estimator (26) follows from the properties of the standard least squares estimator under the assumption that $\theta_{0} \in \mathcal{D}_{\theta}^{\mathrm{qs}}$.

Remark 4. Let us assume that the noise $e(t)$ ranges within a given bound $\Delta e$, i.e., $\|e(t)\| \leq \Delta e$. The feasible parameter set $\mathcal{D}_{\theta}$, i.e., the set of all values of $\theta$ that are consistent with the assumed model structure, the collected input-output data and the assumed error bound $\Delta e$, is known to be a semialgebraic region described by a set of polynomial inequalities (see, e.g., Cerone et al. (2011a)). Thus, estimator (26) can be rewritten by constraining the optimization to the feasible parameter set $\mathcal{D}_{\theta}$, i.e.,

$$
\begin{aligned}
\hat{\theta}= & \arg \min _{\theta, P, \bar{c}} J(\theta, \bar{u}, \bar{y}) \\
\text { s.t. } & \bar{c}^{\top} \bar{a}\left(\lambda_{i}^{v}\right)+\bar{a}\left(\lambda_{i}^{v}\right)^{\top} \bar{c}-F(P) \succeq 0, \forall i=1,2, \ldots, \rho \\
& \theta \in \mathcal{D}_{\theta} \\
& C \in \mathcal{S}_{c} .
\end{aligned}
$$

Since $\mathcal{D}_{\theta}$ is described by polynomial inequalities, problem (27) is a PMI problem of the same kind as (26).

By exploiting the generalization of the Descartes' rule (Basu et al. (2006)), it is possible to apply to (26) and (27) the socalled scalarization approach, proposed in Lasserre (2005), which allows to exactly replace the PMI constraints $\bar{c}^{\top} \bar{a}\left(\lambda_{i}^{v}\right)+$ $\bar{a}\left(\lambda_{i}^{v}\right)^{\top} \bar{c}-F(P) \succeq 0, \forall i=1,2, \ldots, \rho$ with a set of scalar multivariate polynomial constraints. Define the following matrix

$$
\mathcal{H}_{i}(x)=\bar{c}^{\top} \bar{a}\left(\lambda_{i}^{v}\right)+\bar{a}\left(\lambda_{i}^{v}\right)^{\top} \bar{c}-F(P),
$$

whose entries are scalar polynomials in the variable

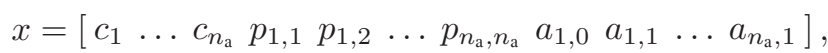

where $p_{i, j}$ is the $(i, j)$-th entry of matrix $P$. The characteristic polynomial $p_{i}(w, x)$ of $\mathcal{H}_{i}(x)$ can be written, without loss of generality, as

$$
\begin{aligned}
p_{i}(w, x) & =\operatorname{det}\left(w I_{n_{\mathrm{a}}+1}-\mathcal{H}_{i}(x)\right) \\
& =w^{n_{\mathrm{a}}+1}+\sum_{k=1}^{n_{\mathrm{a}}+1}(-1)^{k} h_{k}(x) w^{n_{\mathrm{a}}+1-k}, w \in \mathbb{R}
\end{aligned}
$$

where $h_{k}(x)$ are scalar polynomials and $I_{n_{\mathrm{a}}+1}$ denotes the identity matrix of size $n_{\mathrm{a}}+1$. Application of the scalarization approach to matrix $\mathcal{H}_{i}(x)$ leads to the following result.

Result 2. (Lasserre (2005))

$$
\mathcal{H}_{i}(x) \succeq 0 \text { if and only if } h_{k}(x) \geq 0 \forall k=1, \ldots, n_{\mathrm{a}}+1 \text {. }
$$

Due to Result 2, problem (26) can be converted to a standard polynomial optimization problem which can be solved by exploiting the relaxation-based approaches proposed in Lasserre (2001); Parrillo (2003). In particular, by means of the momentbased approach described in Lasserre (2001), a hierarchy of relaxed semidefinite programming (SDP) problems are constructed whose optima are guaranteed to converge to the global optimum of problem (26). An efficient MATLAB implementation of moment-based relaxation techniques can be found in the software Gloptipoly by Henrion and Lasserre (2003).
An alternative approach to solve problem (26) is based on LMI relaxation for PMI problems proposed in Henrion and Lasserre (2006) in the spirit of the results of sum-of-squares decompositions of positive polynomial matrices discussed in Hol and Scherer (2004, 2005). In particular, in Henrion and Lasserre (2006), PMI constraints of the kind $\mathcal{H}_{i}(x) \succeq 0$ are directly handled in matrix form without conversion to a set of scalar polynomials and a sequence of convex SDPrelaxed problems is built up, whose solutions are guaranteed to converge to the global optimum of the original nonconvex PMI problem. Such an approach allows one to detect if the global optimum of the original PMI problem (26) is reached, and if so, to extract the global minimizers. From a computational point of view, this methodology is more convenient than the scalar approach when the degree and the size of polynomial matrix $\mathcal{H}_{i}(x)$ is large (see Henrion and Lasserre (2006) for a detailed discussion).

\section{A SIMULATION EXAMPLE}

In this section, we show the effectiveness of the presented approach through a numerical example. The LPV data-generating system is described by eqs. (1a)-(1c) with $\mathcal{A}\left(q^{-1}, \lambda_{t}\right)=1+$ $\left(-1.41+0.1 \lambda_{t}\right) q^{-1}+\left(0.942+0.04 \lambda_{t}\right) q^{-2}$ and $\mathcal{B}\left(q^{-1}, \lambda_{t}\right)=$ $\left(1.5-1.1 \lambda_{t}\right) q^{-2}$. Therefore, the true parameter vector is $\theta_{0}=$ $\left[a_{1,0}, a_{1,1}, a_{2,0}, a_{2,1} b_{2,0}, b_{2,1}\right]^{\top}=[-1.41,0.1,0.924,0.04$, $1.5,-1.1]^{\top}$. The system is simulated using a white input signal $u_{t}$ with uniform distribution on the interval $[-5 ; 5]$; a white error $e_{t}$ uniformly distributed within $[-4 ; 4]$ and a white scheduling parameter $\lambda_{t}$ with uniform distribution within $[-0.3 ; 0.3]$. It is worth pointing out that the LPV datagenerating system is quadratically stable for all trajectories of the scheduling parameter $\lambda_{t}$ in the interval $[-0.3 ; 0.3]$. As a matter of fact, conditions of Theorem 2 are satisfied for the following stable central polynomial $\mathcal{C}\left(q^{-1}\right)$ :

$$
\mathcal{C}\left(q^{-1}\right)=1-1.4793 q^{-1}+0.9792 q^{-2} .
$$

The LPV model is identified from a set of data collected from $N=100$ measurements. Under these conditions, the signal to noise ratio in the generated data, defined as

$$
\mathrm{SNR}_{w}=10 \log _{10} \frac{\left\|w_{t}\right\|_{2}^{2}}{\left\|\eta_{t}\right\|_{2}^{2}}
$$

is $7 \mathrm{~dB}$.

First, a set of parameters $\hat{\theta}_{\mathrm{NS}}$ is computed by minimizing the standard LS functional $J=\|\bar{y}-\Phi \theta\|_{2}^{2}$ given in (8) without enforcing stability constraints. The obtained $\hat{\theta}_{\mathrm{NS}}$ is reported in Table 2. It is worth noting that the identified LPV model is not stable for all the trajectories of the scheduling parameter $\lambda_{t}$ within $[-0.3 ; 0.3]$. In fact, when $\lambda_{t}=-0.3$ for all $t=1,2, \ldots$, the LPV system is reduced to an LTI model with a $z$-transfer function

$$
\frac{1.529}{z^{2}-1.534 z+1.01}
$$

which shows poles outside the unit circle. In order to ensure stability of the identified model, LPV parameters $\hat{\theta}$ are then evaluated as solution of constrained problem (26). The same LS functional $J$ previously employed to compute parameters $\hat{\theta}_{\mathrm{NS}}$ is used as objective function in (26). In order to solve the nonconvex PMI problem (26), the relaxation approach proposed by Henrion and Lasserre (2006) has been implemented in the Yalmip Matlab interface (Löfberg (2004)). The obtained 
Table 2. Comparison of the true parameters $\theta_{0}$, the parameters $\hat{\theta}_{\mathrm{NS}}$ estimated without imposing stability constraints and the parameters $\hat{\theta}$ estimated by enforcing stability constraints.

\begin{tabular}{cccc}
\hline Parameter & $\theta_{0}$ & $\hat{\theta}_{\mathrm{NS}}$ & $\hat{\theta}$ \\
\hline$a_{1,0}$ & -1.4100 & -1.4592 & -1.4444 \\
$a_{1,1}$ & 0.1000 & 0.2507 & 0.0915 \\
$a_{2,0}$ & 0.9240 & 0.9570 & 0.9420 \\
$a_{2,1}$ & 0.0400 & -0.1761 & 0.0204 \\
$b_{2,0}$ & 1.5000 & 1.5291 & 1.5068 \\
$b_{2,1}$ & -1.1000 & -2.2024 & -1.9893 \\
\hline
\end{tabular}

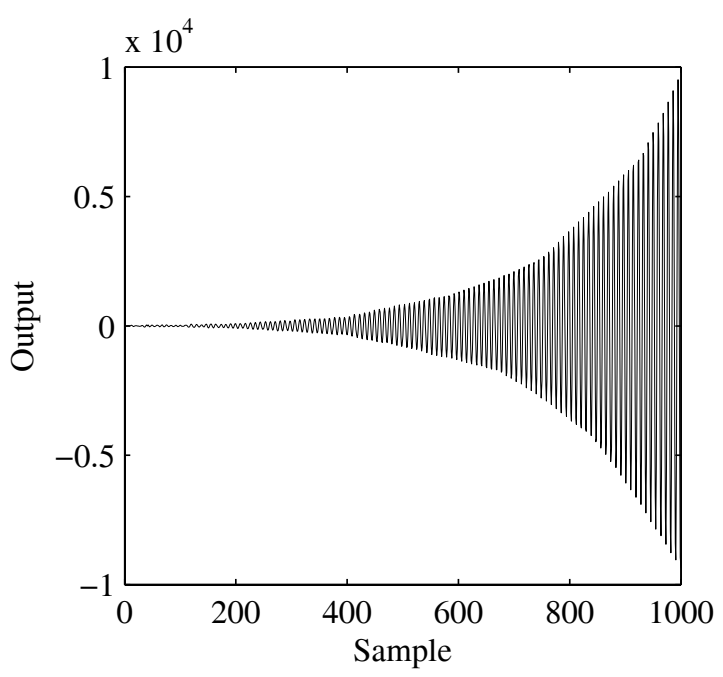

Fig. 1. Output signal $\hat{y}_{t}^{\mathrm{NS}}$ obtained by the LPV model associated with the parameter vector $\hat{\theta}_{\mathrm{NS}}$ computed without enforcing stability constraints.

values of $\hat{\theta}$ are reported in Table 2. By exploiting the global optimality check presented in Henrion and Lasserre (2006), the obtained values of $\hat{\theta}$ are proven to be the global minimizer of the nonconvex problem (26). This means that the identified LPV model is guaranteed to be quadratically stable for all trajectories of the scheduling variable $\lambda_{t}$ in the interval $[-0.3 ; 0.3]$. The elapsed time to compute $\hat{\theta}$ is about 10 seconds on a $2.40-\mathrm{GHz}$ Intel Pentium IV with 3 GB of RAM. Performance of the estimated LPV model is tested on a validation set, where data are collected under a scheduling parameter trajectory $\lambda_{t}=-0.3$ for all $t=1,2, \ldots$, which is a scheduling trajectory that destabilizes the LPV model associated with $\hat{\theta}_{\mathrm{NS}}$. The output $\hat{y}_{t}^{\mathrm{NS}}$ of the model associated with the parameter vector $\hat{\theta}_{\mathrm{NS}}$ is reported in Fig. 1, showing an unstable behaviour of the model identified without enforcing stability constraints. In Fig. 2, only the first 100 samples of the estimated output $\hat{y}_{t}^{\text {NS }}$ are reported, and then compared to the output signal $y_{t}$ of the real system and to the estimated output $\hat{y}_{t}$ of the identified stable LPV model associated with the parameter vector $\hat{\theta}$. The error $\xi_{t}=y_{t}-\hat{y}_{t}$ between the true output signal $y_{t}$ and the estimated output $\hat{y}_{t}$ is plotted in Fig. 3, together with the error $\xi_{t}^{\mathrm{NS}}=y_{t}-\hat{y}_{t}^{\mathrm{NS}}$ between $y_{t}$ and the estimated output $\hat{y}_{t}^{\mathrm{NS}}$. In order to evaluate the performance of the identified models, the best fit rate (BFR) and the mean square error (MSE), defined as follows, are computed on the first 100 samples of the validation data set:

$$
\mathrm{BFR}=\max \left(1-\frac{\left\|y_{t}-\tilde{y}_{t}\right\|_{2}}{\left\|y_{t}-\bar{y}_{t}\right\|_{2}}, 0\right) \cdot 100 \%,
$$

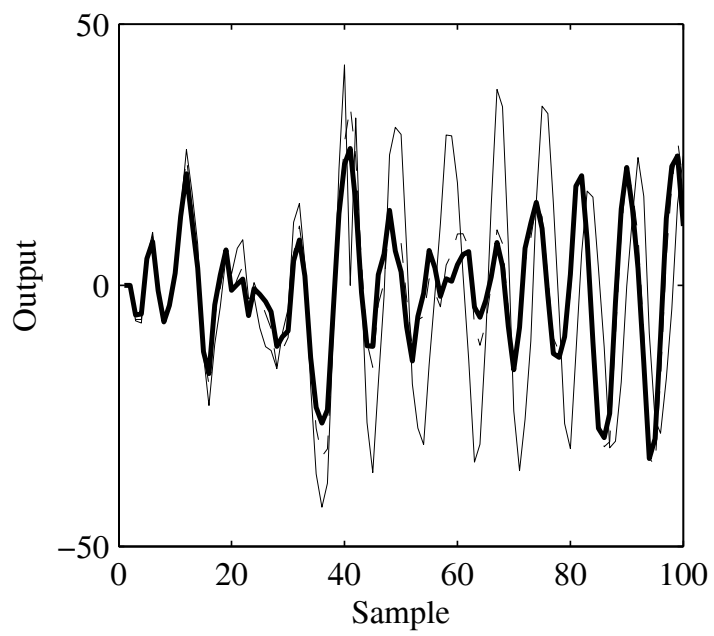

Fig. 2. Output signal $y_{t}$ generated by the true LPV system (solid thick line), estimated output signal $\hat{y}_{t}$ obtained by the identified stable LPV model (dashed thin line) and output signal $\hat{y}_{t}^{\mathrm{NS}}$ obtained by the LPV model identified without enforcing stability constraints (solid thin line).

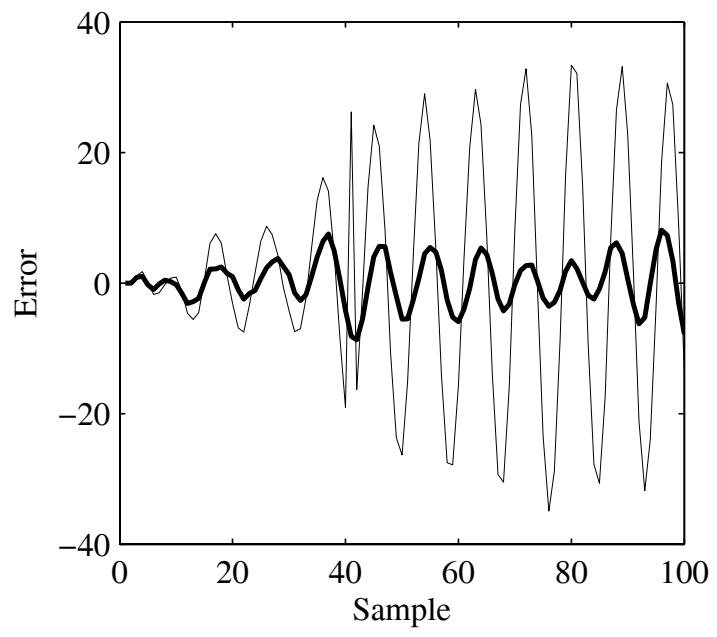

Fig. 3. Error $\xi_{t}=y_{t}-\hat{y}_{t}$ between the true output signal $y_{t}$ and the output signal $\hat{y}_{t}$ (solid thick line) and error $\xi_{t}^{\mathrm{NS}}=y_{t}-$ $\hat{y}_{t}^{\mathrm{NS}}$ between the true output signal $y_{t}$ and the output signal $\hat{y}_{t}^{\mathrm{NS}}$ (solid thick line).

Table 3. Best fit rate (BFR) and mean square error (MSE) computed for the LPV models associated with parameters $\hat{\theta}_{\mathrm{NS}}$ and $\hat{\theta}$.

\begin{tabular}{ccc}
\hline $\begin{array}{c}\text { Model } \\
\text { parameters }\end{array}$ & BFR & MSE \\
\hline$\hat{\theta}_{\mathrm{NS}}$ & $0 \%$ & 1.73 \\
$\hat{\theta}$ & $71 \%$ & 0.32 \\
\hline MSE $=\frac{1}{100}\left\|y_{t}-\tilde{y}_{t}\right\|_{2}^{2}$,
\end{tabular}

where $\bar{y}_{t}$ is the mean value of $y_{t}$, while $\tilde{y}_{t}$ is either $\hat{y}_{t}$ or $\hat{y}_{t}^{\mathrm{NS}}$. The computed values of BFR and MSE are reported in Table 3. Indeed, the obtained results show that the proposed method, based on stability constraints enforcement, significantly outperforms the standard least squares approach. 


\section{CONCLUSION}

The problem of enforcing stability constraints in the identification of LPV models is addressed in the paper. In particular, a systematic approach is proposed to force quadratic stability for the case of input-output LPV models with affine dependence on a vector-valued scheduling variable assumed to range inside a polytope. The problem is formulated in terms of PMI optimization and solved exploiting suitable semidefinite relaxation techniques. Applicability of the proposed approach in the presence of either stochastic or deterministic bounded equation error is briefly discussed. The effectiveness of the proposed approach is shown by means of a simulation example where application of standard least squares estimation leads to an unstable model.

\section{ACKNOWLEDGMENTS}

This research was mainly developed when Dr. D. Piga was a Ph.D student at the Politecnico di Torino and concluded when he was a postdoc at the Delft University of Technology.

\section{REFERENCES}

Bamieh, B.A. and Giarré, L. (2002). Identification of linear parameter-varying models. International Journal of Robust Nonlinear Control, 12(9), 841-853.

Basu, S., Pollack, R., and Roy, M.F. (2006). Algorithms in real algebraic geometry. Springer-Verlag New York Inc.

Cerone, V., Piga, D., and Regruto, D. (2011a). Convex relaxation techniques for set-membership identification of LPV systems. In Proc. of the American Control Conference 2011, 171-176. San Francisco, California (USA).

Cerone, V., Piga, D., and Regruto, D. (2011b). Enforcing stability constraints in set-membership identification of linear dynamic systems. Automatica, 47(11), 2488-2494.

Cerone, V. and Regruto, D. (2008). Set-membership identification of LPV models with uncertain measurements of the time-varying parameter. In Proc. of the 47th IEEE Conference on Decision and Control, 4491-4496.

Felici, F., Van Wingerden, J.W., and Verhaegen, M. (2007). Subspace identification of MIMO LPV systems using a periodic scheduling sequence. Automatica, 43, 1684-1697.

Gilbert, W., Henrion, D., Bernussou, J., and Boyer, D. (2010). Polynomial LPV synthesis applied to turbofan engines. Control Engineering Practice, 18, 1077-1083.

Henrion, D. and Lasserre, J.B. (2003). Gloptipoly: Global optimization over polynomials with Matlab and SeDuMi. ACM Transactions Math. Soft., 29, 165-194.

Henrion, D. and Lasserre, J.B. (2006). Convergent relaxations of polynomial matrix inqequalities and static output feedback. IEEE Transactions on Automatic Control, 51(2), 192 202.

Henrion, D., Sebek, D., and Kucera, V. (2003). Positive polynomials and robust stabilization with fixed-order controllers. IEEE Trans. on Automatic Control, 48(7), 1178-1186.

Hol, C.W.J. and Scherer, C.W. (2004). Sum of squares relaxations for polynomial semidefinite programming. In Proc. Symp. Mathematical Theory of Networks and Systems (MTNS), Leuven, Belgium, 2004.

Hol, C.W.J. and Scherer, C.W. (2005). A sum of squares approach to fixed-order $H_{\infty}$ synthesis. 580-580.

Janakiraman, P. and Bhattacharyya, A. (1999). Stable output error identification scheme using periodic excitation signals. In Proc. of IEEE International Symposium on Circuits and Systems, volume 5, 539-542. Orlando, Florida (USA).
Lacy, S. and Bernstein, D. (2003). Subspace identification with guaranteed stability using constrained optimization. IEEE Trans. Automatic Control, 48(7), 1259-1263.

Lasserre, J.B. (2001). Global optimization with polynomials and the problem of moments. SIAM Journal on Optimization, $11,796-817$.

Lasserre, J.B. (2005). A unified criterion for positive definiteness and semidefiniteness. LAAS-CNRS, Toulouse, France, Res. Rep. 05283.

Laurain, V., Gilson, M., Tóth, R., and Garnier, H. (2010). Refined instrumental variable methods for identification of LPV Box-Jenkins models. Automatica, 46(6), 959-967.

Löfberg, J. (2004). YALMIP: A toolbox for modeling and optimization in Matlab. In IEEE International Symposium on Computer Aided Control Systems Design, 284-289. Taipei, Taiwan.

Lopes dos Santos, P., Ramos, J.A., and Martins de Carvalho, J.L. (2008). Subspace identification of linear parametervarying systems with innovation-type noise models driven by general inputs and a measurable white noise time-varying parameter vector. Int. J. of Systems Science, 39(9), 897-911.

Nicolao, G.D. and Pillonetto, G. (2008). A new kernel-based approach for system identification. In Proc. of the American Control Conference, 4510-4516. Seattle, Washington (USA).

Parrillo, P. (2003). Semidefinite programming relaxations for semialgebraic problems. Mathematical Programming, 96, 293-320.

Previdi, F. and Lovera, M. (2004). Identification of non-linear parametrically varying models using separable least squares. International Journal of Control, 77(16), 1382-1392.

Regalia, P. and Stoica, P. (1995). Stability of multivariable least-squares models. IEEE Signal. Processing Letters, 2(10), 195-196.

Rugh, W.J. and Shamma, J.S. (2000). Research on gain scheduling. Automatica, 36(10), 1401-1425.

Scherer, C. (2006). LMI relaxations in robust control. European Journal of Control, 12(1), 3-29.

Tóth, R. (2010). Modeling and identification of linear parameter-varying systems. Lecture Notes in Control and Information Sciences, 403. Springer Germany.

Tóth, R., Heuberger, P.S.C., and Van den Hof, P.M.J. (2010). Asymptotically optimal orthonormal basis functions for LPV system identification. Automatica, 45(6), 1359-1370.

Tugnait, J. and Tontiruttananon, C. (1998). Identification of linear systems via spectral analysis given time-domain data: Consistency, reduced-order approximation, and performance analysis. IEEE Trans. Automatic Control, 43(10), 13541373.

Verdult, V. and Verhaegen, M. (2002). Subspace identification of multivariable linear parameter-varying systems. Automatica, 38, 805-814.

Verdult, V. and Verhaegen, M. (2005). Kernel methods for subspace identification of multivariable LPV and bilinear systems. Automatica, 41, 1557-1565. 